\title{
An Application of Jacobian Matrix in Kinematic of Multi-loop Spatial Mechanism
}

\author{
Kaushik Patel, Pina M Bhatt
}

\begin{abstract}
Mechanism is an intended to turn input motion into a required set of output motion and forces. Now a days the spatial mechanism is widely used in various industrial applications like parallel kinematic machines as a robot manipulator. The main aim of the review paper is to use the Jacobian matrix into the kinematic analysis. From the review papers analysis, it has been concluded that the Kinematic analysiswith help of Jacobian matrix performed various Multi-loop Spatial Mechanism settings for Kinematic geometry, its topology and to carried out displacement modelling. It also used to Kinematic Design, synthesis and optimization. Finally, Comparison of existing methods for Kinematic Singularities with Jacobian in Parallel Manipulator.
\end{abstract}

Keywords: Kinematic analysis, multi loop spatial mechanism, Jacobian matrix

\section{INTRODUCTION}

$\mathrm{P}_{\mathrm{a}}$ arallel kinematic machines (PKM) offer numerous advantages, including high position accuracy, high inherent static / dynamic rigidity, and low inertia. High nominal load-to-weight ratio and outstanding dynamic efficiency give benefit and drawbacks for PKMs. are tiny and complex workspace, frequently paired position and moving platform orientation as well as complicated forward position kinematics.

Assessment of Kinematic spatial mechanism using distinct principles based on their capacity to use distinct approaches, criteria or techniques for a distinct mechanism type. Researchers reviewed the multi-loop spatial mechanism design and dynamic balancing [1]. Different kinds of input and output desirable relation established with synthesis in the spatial multi-loop system.

\section{Kinematic ANALYSIS}

Different types of methods, concepts, criteria or techniques used to carry out kinematic analysis of spatial mechanisms with a single loop to multi-loop with mobility Kinematic analysis carried out for different configurations of Multi-loop spatial mechanism to carry out Displacement Summary, 3-d Notation Simulation to obtain Work- space volume, Singularity observation etc. For optimal position analysis for RSSR kinematic model can be expanded to RSSR-SS and develop Input -output relationship to link incremental speed parameters.

Revised Manuscript Received on October31, 2019.

* Correspondence Author

Kaushik Patel*, Government Engineering College, Patan, Gujarat Technological University, Gujarat, India. Email: kvpatel@live.in

Dr. Pina M Bhatt, Aditya Silver Oak Institute of Technology, Gujarat Technological University, Gujarat, India.

\author{
A. Kinematic geometry, topology and displacement \\ modeling
}

In Spatial mechanism, a kinematic analysis obtains using line trajectory or line geometry. Al-Ghefari and Abdel-Baky derived Euler-Savary equation and established the relation between axodes and higher-order instantaneous invariants. Velocity and acceleration gained with the second-order motion of spherical and planar [2].

Müller represents the kinematic modelling and establishes a systematic representation of the kinematic topology, which shows the relation between the relation of links and joints. Higher-order kinematic analysis work on loop closure constraints with cut-joint (for higher kinematic pair) and cut-body (for lower pairs) approach. Compare various graphs without matrix formation [3].

Ceccarelli et al. formulate Velocity and acceleration model on extensions of the RSSR and RRSS to RSSR-SS mechanism and work on analysis rather than mechanism synthesis, which is fully analytical displacement/velocity model formulate with rigid body guidance. It's utilized for Function generation with Zero calculation error Vector strategy for calculating the tooltip route and speed profiles in the manipulator of the friction instrument [4]. J. S. Zhao et al. optimized A new technique with a structure parameter in algebra geometry method. It is used to investigate the workspace of manipulator very precisely and efficiently with coordinate transformation using multi translation DOF [5].

Displacement analysis model develops by Russell and Sodhi on an adjustable movement generator of RSSR-SC mechanism. By utilizing the passive degree of freedom. The Newton method is a technique of root-finding to give multiphase motion generation by developing transformation matrices. They use Math CAD software to analysis of spatial five-bar multi-loop mechanisms for a range of link angle, constant length and orthogonally constraints [6].

\section{B. Kinematic design}

Cervantes-Sánchez et al. suggest the kinematic Design with various range of motion in a multi-loop spatial mechanism. Without considering branch defect, a quartic equation gives polynomial discriminants with unidirectional rotating actuator motion for input link angle. Displacement analysis, branching analysis, Reliability conditions carried out by simplified global equation [7]. Multi-loop parallel spatial mechanisms with less liberty solved by the forward and inverse kinematics in a number of distinguishing equations. According to Zhou et al., reciprocal screw theory equation solves by inverse screw that decides the dimension and independent movement [8].

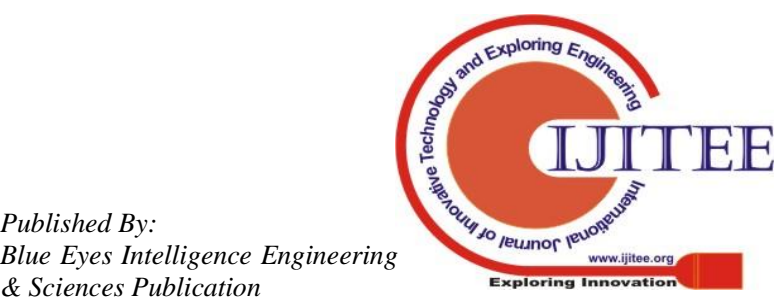


Analysis and synthesis carried out for Adjustable multi-loop spatial mechanism like RSSR-SC design with Multiphase motion generation. RUSSELL and SODHI do not consider the branch and order defect in RSSR-SS mechanism, which is distinct from RSSR-SC [9].

Multi-loop PM also used in sun tracking application in a particular region rather than the polar region with static analysis and Constant Speed. Muralidharan and Bandyopadhyay reduce application cost and energy requirement by detail analysis of loop closer equation in forward and inverse kinematics [10].

\section{Kinematic synthesis and optimization}

Kinematic synthesis deals with forces and movement. In RSSR-SS multi-loop spatial mechanism, the synthesis works on 3 kinds of constraints using Vector approach. Sandor optimizes two closed-loop using Hooke and Jeeves directed patterns search method for 10 design variable ( 3 for each R-S \& 4 for S-S), but it is limited to only four precision point. Crank ratability, fixed pivot location, transmission characteristics can be considered [11]. The Generalized Reduced Gradient method is used to solve equality and inequality constraints. Complexity kinematic analysis of that mathematical equation for constant length solve by help of computer-controlled mechanism

$$
\left(A_{j}-B_{j}\right)^{T}\left(A_{j}-B_{j}\right)=\left(A_{1}-B_{1}\right)^{T}\left(A_{1}-B_{1}\right)
$$

where $\mathrm{Aj}$ is obtained from input angle $\theta \mathrm{j}[12]$.

To synthesize RSSR-SS Adjustable Mechanisms, Sodhi [13] used $\mathrm{r}$ finite and multiplied separated positions for adjustable RSSR-SS mechanism. He Considered issue with fixed crank and follower for to phase fixed pivot adjustment. Limited work carried out to eliminating branch defeat and order defect for that work. Shen et al. optimize Branch defect and order defect in small scale for multi-loop spatial mechanism in motion generation using a nonlinear equation system with Mat lab. It is structurally sound mechanism [14].

\section{KINEMATIC SINGULARITIES AND JACOBIAN IN PARALLEL MANIPULATOR}

Inverse mapping of Cartesian space to joint space in Cartesian space can be referred to as Kinematic Singularities in robot manipulator. There are mainly two types of singularities (i) Boundary Singularities (full extension of a joint) and (ii) Internal Singularities (alignment of the axes of the Robot in space), and these Singularities reduced the mobility of a manipulator. Singular position of the manipulator can perform analysis by Using Jacobian matrix with infinite inverse kinematic solutions.

Unlike serial manipulators, where all the joints are actuate $\mathrm{d}$, only some of the joints are actuated for a parallel manipul ator, the rest being passive.

Goehler and Murray derived altered technique and used software packages such as MatLab to compare with current technique to study the kinematic spatial manipulators rapidly and effectively.

$$
{ }^{n} p=\left[\begin{array}{l}
x \\
y \\
z
\end{array}\right]
$$

Joint velocities described with existing Jacobian matrix, and homogeneous matrix formation derives by

$$
{ }^{0} p={ }_{1}^{0} T{ }_{2}^{1} T \ldots{ }_{n-1}^{n} T^{n} p
$$

and modified Jacobian matrix

$$
{ }^{0} p={ }_{1}^{0} T\left(\theta_{1}\right){ }_{2}^{1} T\left(\theta_{2}\right) \ldots \ldots \cdot{ }_{n-1}^{n} T\left(\theta_{n}\right)^{n} p
$$

With the help of symbolic partial derivation and new modified metrics solve and reduce momentous computation time in MatLab. [15]

Chembrammel and Kesavadas suggest matrix estimation of a Jacobian method. That method used for real-time implementation of kinematics and dynamics in complex planar and spatial robots with changes in the structure [16].

And used Existing Method finds liner and angular velocity with using screw theory.

$$
\omega=\sum_{i=1}^{n} q_{i} z_{i-1} \text { And } v=-\sum_{i=1}^{n}\left(q_{i} z_{i-1}\right) \times p_{i-1}
$$

Joshi and Tsai Analysis the Jacobian for Limited-DOF in Parallel Manipulators. They Develop Jacobian metric for 3-UPU and 3-RPS manipulators in conjunction with the help of reciprocal screw theory to find architectural and constrain singularities for lass then six degrees of freedom [17].

And derive Jacobian for moving platform or end-effector if DOF $<6$, that is

$$
\$_{p}=\sum_{i=1}^{c_{i}} \dot{\theta}_{j, i} \widehat{\$_{j, i}}
$$

For $\mathrm{i}=1,2 \ldots \mathrm{F}$.

Jacobian of Constraints in Eq. (5) and Jacobian of Actuations (2) for (6-Ci) based on reciprocal screws yields with the orthogonal product are

$$
\widehat{\$}_{r, k, i}^{\mathrm{T}} \$_{\mathrm{p}}=0
$$

For $\mathrm{k}=1,2, \ldots 6-\mathrm{Ci}$

$$
\widehat{\$}_{r, 6-C_{i}+1, i}^{T} \$_{p}=\dot{\theta}_{g_{i}, i} \widehat{\$}_{r, 6-c_{i}}^{T}+1, i \widehat{\$}_{g_{i}, i}
$$

And finally matrix $\mathrm{j}_{\mathrm{x}} \$_{\mathrm{p}}=\mathrm{j}_{\mathrm{q}} \dot{\mathrm{q}}$, Although Overall Jacobian of the parallel manipulator is

$\dot{\mathrm{q}}_{\mathrm{o}}=\mathrm{J} \$_{\mathrm{p}}$, Were qo is a $6 \times 1$ and $\mathrm{J}$ is a $6 \times 6$ matrix.

Hoevenaars et al. focused on the inverse kinematic analysis to established relationships and obtained an expression of differentiated Jacobian. They derived Complete Jacobian analysis using screw theory with some assumption like Terminal link, internal serial chain, End-effector serial chain, Virtual joint Connectivity [18]. 


$$
\left[\begin{array}{c}
\dot{\mathrm{q}}_{1} \\
\dot{\mathrm{q}}_{2} \\
\vdots \\
\dot{\mathrm{q}}_{\mathrm{N}}
\end{array}\right]=\left[\begin{array}{c}
\mathrm{J}_{1}^{-1} \\
\mathrm{~J}_{2}^{-1} \\
\cdot \\
\mathrm{J}_{\mathrm{N}}^{-1}
\end{array}\right] \$_{\mathrm{t}, \mathrm{e}}
$$

$$
\left[\begin{array}{c}
\dot{\mathrm{q}}_{1} \\
\dot{\mathrm{q}}_{2} \\
\vdots \\
\dot{\mathrm{q}}_{\mathrm{N}}
\end{array}\right]=\left[\begin{array}{cccc}
\mathrm{J}_{1}^{-1} & 0_{6 \times 6} & \cdots & 0_{6 \times 6} \\
0_{6 \times 6} & \mathrm{~J}_{2}^{-1} & \cdots & 0_{6 \times 6} \\
\vdots & \vdots & \ddots & \vdots \\
0_{6 \times 6} & 0_{6 \times 6} & \cdots & \mathrm{J}_{\mathrm{N}}^{-1}
\end{array}\right]\left[\begin{array}{c}
\$_{\mathrm{t}, 1} \\
\$_{\mathrm{t}, 1} \\
\vdots \\
\$_{\mathrm{t}, \mathrm{N}}
\end{array}\right]
$$

a)

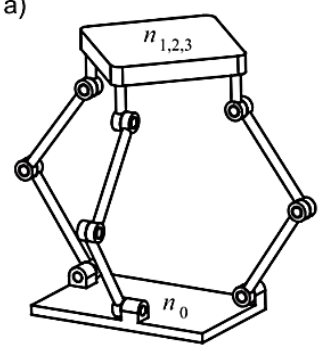

b)

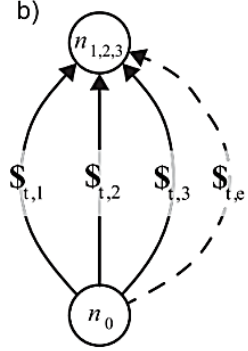

Figure1a) An instance of a traditional three-leeged PM and b) its representation in graph theory [18]

Y. Zhao et al. operate on the Jacobian PM matrix (parallel mechanisms) that usually differs with the poses of the movi ng workspace platform. They identify motion characteristic of proposed the constant Jacobian matrix and work on a continuous Jacobian matrix.

A distinct strategy, like the vector method and the theory of the screw is used for the Jacobian matrix.

$\mathrm{U}=\mathrm{J} \dot{\mathrm{q}}$ Were $\mathrm{U}$ is output velocity and $\dot{\mathrm{q}}$ is input velocity.

Identify requirements for TPM (Translational Parallel Mechanisms), Rotational PM, and Mashed-Motion PM with steady worldwide frame transmission [19].

Rezaei et al. Suggest non-pure transnational Jacobin matrices for the moving platform's connection between angular and transnational speeds. Two inverse kinematics solutions are used for operation mode with direct kinematics of 3-PSP parallel manipulator of the Robot.

They also use 3 unit vectors $\mathrm{u}, \mathrm{v}$ and $\mathrm{w}$ along the $\mathrm{u}, \mathrm{v}$ and $\mathrm{w}$ axis of the moving co-ordinate frame $\{\mathrm{T}\},{ }_{\mathrm{T}}^{\mathrm{B}} \mathrm{R}$

$$
{ }_{\mathrm{T}}^{\mathrm{B}} \mathrm{R}=\mathrm{R}_{\mathrm{z}, \lambda} \mathrm{R}_{\mathrm{y}, \varphi} \mathrm{R}_{\mathrm{x}, \theta}=\left[\begin{array}{lll}
\mathrm{u}_{\mathrm{x}} & \mathrm{v}_{\mathrm{x}} & \mathrm{w}_{\mathrm{x}} \\
\mathrm{u}_{\mathrm{y}} & \mathrm{v}_{\mathrm{y}} & \mathrm{w}_{\mathrm{y}} \\
\mathrm{u}_{\mathrm{z}} & \mathrm{v}_{\mathrm{z}} & \mathrm{w}_{\mathrm{z}}
\end{array}\right]
$$

And derive Inverse position analysis of end effectors with Jacobian.

$$
\dot{\mathrm{q}}=\mathrm{J} \dot{\mathrm{x}} \text { Where } \mathrm{J}=\mathrm{J}_{\text {inv }}^{-1} \mathrm{~J}_{\text {dir }}
$$

is overall Jacobian matrix.

In addition, the vectors, reference frames, movement variables and DOFs descriptions are provided [20].

Yang et al. [21] used the streamlined the method for calculating the mobility of all types of parallel systems only with Jacobian matrixes and developed a relationship between DOF and in changing platform with output speeds.

The degree of liberty for simple parallel systems is calculated by

$$
\left[\begin{array}{ccc}
\mathrm{J}_{\mathrm{A}} & -\mathrm{J}_{\mathrm{B}} & 0 \\
\mathrm{~J}_{\mathrm{A}} & 0 & -\mathrm{J}_{\mathrm{C}}
\end{array}\right] \dot{\mathrm{Q}}=0
$$

Were JA, JB, and JC respectively Jacobian chain matrix A, $\mathrm{B}$, and $\mathrm{C}$. $\mathrm{Q}$ is the component of $\mathrm{N} \cdot 1$. Calculating the Jacobian matrix and mobility is the primary drawback.

Choi and Ryu compared and discuss the $6 \times 6$ and $4 \times 4$ Jacobian matrices and suggested to expanding $6 \mathrm{x}$ 6 Jacobian matrices for $\mathrm{H} 4$ parallel manipulator (3T and $1 \mathrm{R}$ vertical axis). From the expansion of the Jacobian matrix for 4 DOF [22], three distinct restricted singularities can be acquired.

$$
\begin{gathered}
\$_{\mathrm{t}}={ }^{i} \dot{\theta}_{1}{ }^{\mathrm{i}} \hat{\$}_{1}+{ }^{i} \dot{\theta}_{2}{ }^{\mathrm{i}} \hat{\$}_{2}+{ }^{i} \dot{\theta}_{3}{ }^{\mathrm{i}} \hat{\$}_{3}+{ }^{i} \dot{\theta}_{4}{ }^{\mathrm{i}} \hat{\$}_{4}+{ }^{i} \dot{\theta}_{5}{ }^{\mathrm{i}} \hat{\$}_{5} \\
\text { For }(\mathrm{i}=1 \ldots 4)
\end{gathered}
$$

Huang et al. proposed to use screw theory and linear algebra to analyze lower mobility manipulators.

Wrench subspace and twist subspace of actuator with coefficient/intensity can be solved. Also established a relationship for first-order kinematic and static modelling to produce for velocity, accuracy, force and stiffness In less than six degrees of freedom (DOF)[23].

$$
\mathrm{J}_{\mathrm{x}} \mathrm{S}_{\mathrm{t}}=\mathrm{J}_{\mathrm{p}} \delta_{\rho}
$$

$$
\mathrm{J}_{\mathrm{x}}=\left[\begin{array}{l}
\mathrm{J}_{\mathrm{xa}} \\
\mathrm{J}_{\mathrm{xc}}
\end{array}\right], \mathrm{J}_{\rho}=\left[\begin{array}{ll}
\mathrm{J}_{\rho \mathrm{a}} & \\
& \mathrm{J}_{\rho \mathrm{c}}
\end{array}\right], \delta \rho=\left(\begin{array}{l}
\delta \rho_{\mathrm{a}} \\
\delta \rho_{\mathrm{c}}
\end{array}\right)
$$

Jx is $\left(\mathrm{f}+\sum_{\mathrm{i}=1}^{1}\left(6-\mathrm{n}_{\mathrm{i}}\right) \times 6\right)$ matrix and

$J \rho$ is $\quad\left(\sum_{i=1}^{1}\left(6-n_{i}\right)+f\right) \times\left(\sum_{i=1}^{1}\left(6-n_{i}\right)+f\right)$ diagonal matrix with $\mathrm{J} \rho$ no singular.

And generalized Jacobian of parallel manipulators with $2 \leq \mathrm{f} \leq 6 \mathrm{DOF}$ is

$$
\mathrm{J}=\mathrm{J}_{\rho}^{-1} \mathrm{~J}_{\mathrm{x}}=\left[\begin{array}{ll}
\mathrm{J}_{\mathrm{a}}^{\mathrm{T}} & \mathrm{J}_{\mathrm{c}}^{\mathrm{T}}
\end{array}\right]^{\mathrm{T}}
$$

\section{Result ANd Discussion}

The kinematic analysis of manipulator with Jacobian is an expanding area of research. By passive utilization DOF of the coupler with constant length, the present chapter includes the systematic in-depth literature review with a timeline related to kinematics of spatial mechanism. Several scientists have done extensive research on spatial mechanisms, but the present work shows the work of other researchers in the era of the kinematics of spatial mechanism. Here attempts are made to find out and identify the scope of work spatially for spatial mechanism.

1) The customized technique for specific kinematic analyses to manipulate the Jacobian matrix for the manipulator. 
2) Enhanced performance of programming for complex spatial manipulators.

3) By using matrix differential calculus, calculate the Jacobian of robotic manipulators.

4) Reconfigure the robots and reduce the real-time for calculation of Jacobian

5) The concept of the reciprocal screws may beobtained from the $6 x 6$ Jacobian matrix for restricted Degree of freedom.

6) Novel balancing technique employed a constant Jacobian matrix due to its less computing time.

7) Generalized Jacobian is proposed for accuracy, kinestatic and stiffness modeling for few DOF.

\section{FUTURE SCOPE}

The RSSR-SS mechanism having the more significant benefits over the other multi-loop mechanisms as it is a simple design, most notable for motion generation, no prismatic or cylindrical joint, it doesn't have a passive DOF like RSSR, so it can be used for rigid body guidance. And no rigid joint axis and link length like Bennet linkages, and no complications at ground link like RRSC and RSSR-SC. With using generalized in kinematic analysis accuracy, kinestatic and stiffness modeling for few DOF can be obtained with less time in computing using Matlab tool.

\section{REFERENCES}

1. K. V. Patel and P. M. Bhatt, "Design and dynamic balancing of multi-loop spatial mechanism-A Review," Int. J. Res. Eng. Appl. Manag., vol. V, no. 10, pp. 433-438, 2019.

2. R. A. Al-Ghefari and R. A. Abdel-Baky, "Kinematic geometry of a line trajectory in spatial motion," J. Mech. Sci. Technol., vol. 29, no. 9, pp. 3597-3608, 2015.

3. A. Müller, "Representation of the kinematic topology of mechanisms for kinematic analysis," Mech. Sci., vol. 6, no. 2, pp. 137-146, 2015.

4. S. Editor and M. Ceccarelli, Advances in Mechanisms Design, vol. 8 2012.

5. J. S. Zhao, Z. J. Feng, and K. Zhou, "On the workspace of spatial parallel manipulator with multi-translational degrees of freedom," Int. J. Adv. Manuf. Technol., vol. 27, no. 1-2, pp. 112-118, 2005.

6. K. Russell and R. A. J. S. Sodhi, "a a,” vol. 28, no. 2, 2004

7. J. J. Cervantes-Sánchez, M. A. Moreno-Báez, L. A. Aguilera-Cortés, and E. J. González-Galván, "Kinematic design of the RSSR-SC spatial linkage based on rotatability conditions," Mech. Mach. Theory, vol. 40, no. 10 , pp. 1126-1144, 2005.

8. K. Zhou, J. S. Zhao, Z. Y. Tan, and D. Z. Mao, "The kinematics study of a class of spatial parallel mechanism with fewer degrees of freedom," Int. J. Adv. Manuf. Technol., vol. 25, no. 9-10, pp. 972-978, 2005.

9. K. RUSSELL and R. SODHI, "Design of Adjustable of RSSR-SC Mechanisms for Multi-Phase Motion Generation,” JSME Int. J. Ser. C, vol. 48, no. 4, pp. 668-673, 2006.

10. V. Muralidharan and S. Bandyopadhyay, "A Two-Degree-of-Freedom RSSR-SSR Manipulator for Sun-Tracking," Springer Singapore, 2019.

11. G. N. Sandor, "Computer-aided synthesis of two-closed-loop RSSR-SS spatial motion generator with branching and sequence constraints," Mech. Mach. Theory, vol. 21, no. 4, pp. 345-350, Jan. 1986.

12. P. Premkumar and S. Kramer, "Synthesis of Multi-Loop Spatial Mechanisms by Iterative Analysis: The RSSR-SS Path Generator," J. Mech. Des., vol. 112, no. 1, p. 69, 2008.

13. K. R. and R. S. Sodhi, "Kinematic Synthesis of Adjustable RSSR-SS Mechanisms for Multi-Phase Finite and Multiply Separated Positions," J. Mech. Des., vol. 125, no. 4, pp. 847-853, 2004.

14. Q. Shen, W. Lee, and K. Russell, “A small-scale optimization model for RSSR-SS motion generation with branch and order defect elimination in Matlab," vol. 8, no. 3, pp. 1-8, 2014.

15. C. M. Goehler and W. M. Murray, "Computational development of Jacobian matrices for complex spatial manipulators," Adv. Eng. Softw., vol. 47, no. 1, pp. 160-163, 2012.
16. P. Chembrammel and T. Kesavadas, "A new implementation for online calculation of manipulator Jacobian," PLoS One, vol. 14, no. 2, pp. 1-15, 2019.

17. S. A. Joshi and L.-W. Tsai, "Jacobian Analysis of Limited-DOF Parallel Manipulators,” J. Mech. Des., vol. 124, no. 2, p. 254, 2002.

18. A. G. L. Hoevenaars, C. Gosselin, P. Lambert, and J. L. Herder, "A Systematic Approach for the Jacobian Analysis of Parallel Manipulators with Two End-Effectors," Mech. Mach. Theory, vol. 109, no. December 2016, pp. 171-194, 2017.

19. Y. Zhao, Y. Cao, X. Kong, and T. Zhao, "Type Synthesis of Parallel Mechanisms With a Constant Jacobian Matrix,” J. Mech. Robot., vol. 10, no. 6, p. 061011, 2018.

20. A. Rezaei, A. Akbarzadeh, P. M. Nia, and M. R. Akbarzadeh-T, "Position, Jacobian and workspace analysis of a 3-PSP spatial parallel manipulator," Robot. Comput. Integr. Manuf., vol. 29, no. 4, pp. 158-173, 2013.

21. D. C. Yang, J. Xiong, and X. D. Yang, “A simple method to calculate mobility with Jacobian," Mech. Mach. Theory, vol. 43, no. 9, pp 1175-1185, 2008.

22. H. B. Choi and J. Ryu, "Singularity analysis of a four degree-of-freedom parallel manipulator based on an expanded 6 ?? 6 Jacobian matrix," Mech. Mach. Theory, vol. 57, pp. 51-61, 2012.

23. T. Huang, H. T. Liu, and D. G. Chetwynd, "Generalized Jacobian analysis of lower mobility manipulators," Mech. Mach. Theory, vol. 46 , no. 6, pp. 831-844, 2011.

\section{AUTHORS PROFILE}

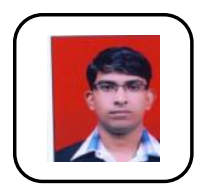

Prof. K. V. Patel is always committed to nurturing and educating students to become lifelong learners. He works as Assistant Professor and Coordinator of the Center of Excellence (Automation) at the Government Engineering College, Patan, Gujarat, India, He has more than 10 years of academic as well as administrative experience. Eight National and International Papers were published in the renowned Journal as well as research papers presented at various conferences. And also, as per GTU Syllabus, he writes four books with different Title and subjects in mechanical engineering as a co-author.

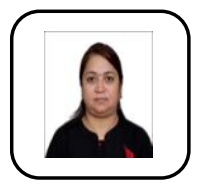

Dr Pina Bhatt with a vision of outcome base education currently working as a Principal of Aditya Silver Oak Institute of Technology, Ahmedabad, Gujarat. She has more than 20 years of Academic and Administrative Experience. During her tenure at GTU and SVBIT she has established two center of Excellence of welding and I-os respectively. Dr Pina Bhatt has awarded Ph D in Mechanical Engineering from RK University and her research was based on Hard Coating for various Industrial Applications. She got Master of Engineering Degree from Gujarat Technological University in the Field of Cryogenic and Bachelor of Engineering from North Gujarat University. She has published more than 15 National and International Papers in reputed Journal as well as presented research papers in various Conference. She is Students Chair of Western India Chapter of AHSRAE and also served as Chapter Working Committee of Indian Society of Heating Refrigeration and Air Conditioning Ahmedabad Chapter for more than three years. She is also active member of IIW, ICC and ASM. profile which contains their education details, their publications, research work, membership, achievements, with photo that will be maximum 200-400 words. 